Cahiers de la recherche sur les droits

Cahiers

${ }^{\text {sur les }}$ Droits fondamentaux

$4 \mid 2005$

Quel avenir pour la laïcité cent ans après la loi de 1905 ?

\title{
La religion à l'école en Angleterre, entre enseignement obligatoire et liberté d'expression
}

Michèle Breuillard

\section{CpenEdition}

\section{Journals}

Édition électronique

URL : https://journals.openedition.org/crdf/7343

DOI : $10.4000 /$ crdf.7343

ISSN : 2264-1246

Éditeur

Presses universitaires de Caen

Édition imprimée

Date de publication : 1 décembre 2005

Pagination : 129-138

ISBN : 2-84133-250-0

ISSN : $1634-8842$

Référence électronique

Michèle Breuillard, « La religion à l'école en Angleterre, entre enseignement obligatoire et liberté

d'expression », Cahiers de la recherche sur les droits fondamentaux [En ligne], 4 | 2005, mis en ligne le 15 décembre 2020, consulté le 14 novembre 2022. URL : http://journals.openedition.org/crdf/7343 ; DOI : https://doi.org/10.4000/crdf.7343 


\title{
La religion à l'école en Angleterre, entre enseignement obligatoire et liberté d'expression
}

\author{
Michèle BREUILLARD
}

Docteur en droit public

Ingénieur d'études CNRS

Centre d'études et de recherches administratives, politiques et sociales

(UMR 8026 CNRS - Université du droit et de la santé, Lille II)

Introduction

I. Les rapports entre l'État, les religions et le service public de l'enseignement

A. Multinationalisme et religions établies

B. La liberté religieuse, principe unique garanti par l'État

C. Enseignement public et liberté d'enseignement

II. Le « modèle " anglais : de l'enseignement religieux à l'étude des religions
A. L'obligation de dispenser un enseignement religieux
B. Le compromis de 1944 : le service public diversifié
C. De l'enseignement religieux à l'enseignement des valeurs chrétiennes

III. L'affaire Shabina Begum, défense et illustration du « modèle anglais "

Conclusion

\section{Introduction}

Ironie du sort ou signe de la prégnance de la question religieuse dans nos sociétés contemporaines? Au moment où la France fête les cents ans de la loi de séparation de l'Église et de l'État, l'actualité politique et judiciaire britannique s'est chargée de relancer le débat sur la place des religions à l'école. D'une part la campagne électorale pour les élections législatives de mai 2005 a remis en débat la place des Églises dans la société britannique. D'autre part, c'est en mars 2005 que rebondit en appel une affaire largement médiatisée opposant une élève musulmane, dont l'avocate n'est autre que l'épouse du Premier Ministre en exercice, à son établissement secondaire, un lycée public de Luton (comté du Bedfordshire).

D’une façon plus large, les récents débats autour de l'héritage chrétien de la civilisation européenne, soulevés à propos du projet de constitution européenne, ont mis en évidence l'isolement des Français par rapport à la majorité de leurs partenaires, notamment nordiques, qu'on ne peut soupçonner de bigoterie. À la différence de la France, État laïque défini par Jean Baubérot comme un «État neutre entre les cultes, indépendants de tous les clergés, dégagés de toute conception théologique ${ }^{1}$, ces pays nordiques auxquels on peut adjoindre la Grande-Bretagne sont, selon Jean Baubérot, des « États laïcocéphales [...] où l’Église

1. F. Buisson, article "Laïcité », in Dictionnaire de pédagogie, cité par J. Baubérot, « Géographie de la laïcité », in Religion et Géographie (Actes du Festival international de géographie, Saint-Dié-des-Vosges, 2002), G. Dorel (dir.), p. 4 (disponible sur le site: http://fig-st-die.education.fr/actes/actes_2002/ bauberot/article. htm). 
nationale se trouve dirigée par un laïc $»^{2}$, en l'espèce le monarque. Le même auteur démontre que la laïcité n'est pas une «exception française ${ }^{3}$ mais pour Olivier Dord, c'est un «modèle sous influence européenne qui s'exporte $\mathrm{mal} »^{4}$. Nous allons tenter de comprendre pourquoi.

Il nous a paru intéressant, en effet, à l'occasion d'une affaire soulevée par le port d'une robe islamique à l'école - et non d'un simple foulard - d'examiner en profondeur le « modèle » anglais (I) qui résulte de rapports différents entre l'État, les religions et le service public de l'enseignement, modèle qu'on peut dire radicalement opposé au «modèle» français et qui a évolué de l'enseignement religieux à l'étude des religions (II). Nous en déduisons que l'affaire Shabina Begum est une parfaite défense et illustration de ce «modèle» anglais (III).

\section{Les rapports entre l'État, les religions et le service public de l'enseignement}

Si la conception française de la laïcité fonde, selon Olivier Dord, «un modèle original au sein des États membres de l'Union européenne ${ }^{5}$, les religions jouissent en Grande-Bretagne d'un statut tout aussi spécifique, qu'on ne retrouve qu'aux États-Unis, au Canada et dans le Commonwealth, même si la Constitution des États-Unis affirme le principe de la séparation des Églises et de l'État. L'expression d' " union dans la diversité » qu'Olivier Dord ${ }^{6}$ applique aux rapports entre l'État et les Églises de Grande-Bretagne correspond tout aussi bien à la situation constitutionnelle du Royaume-Uni dont ils sont l'illustration. Ainsi la Grande-Bretagne s'oppose radicalement à la France sur le sujet qui nous intéresse ici. D’une part, le multinationalisme est une donnée fondamentale des institutions politiques qui se traduit par une pluralité de religions établies (A) et loin de la conception française du droit des cultes, seule est jugée nécessaire l'affirmation d'un principe unique de liberté de pensée et d'expression religieuse (B). D'autre part, si la liberté d'enseignement scolaire est entière comme en France, elle aboutit à une séparation étanche entre un enseignement privé et un enseignement public (C).

\section{A. Multinationalisme et religions établies}

Le Royaume-Uni reconnaît l'existence en son sein de quatre nations, l'Angleterre, l'Écosse, le Pays de Gal- les et l'Irlande du Nord, dont les situations politiques et administratives ont, de tout temps, été particulières. La réforme de la dévolution de 1998-1999 n'a rien simplifié puisque des niveaux différents d'autonomie ont été accordés aux trois régions celtes. Mais dans les trois cas, le contrôle des collectivités locales est une compétence dévolue aux exécutifs régionaux et leur assemblée élue, le Parlement d'Écosse (Scottish Parliament), l'Assemblée nationale galloise (National Assembly for Wales) et l'Assemblée nationale d'Irlande du Nord (Northern Ireland Assembly $)^{7}$. Au contraire, la régionalisation imaginée par le Gouvernement Blair à l'intérieur de l'Angleterre est en panne depuis le rejet du référendum proposé aux citoyens du Nord-Est en novembre 2004, et dans l'attente de leur élection au suffrage universel, les assemblées régionales anglaises fonctionnent actuellement sur un mode plus proche de celui des comités économiques et sociaux que de celui des conseils régionaux français ${ }^{8}$.

Comme nous le verrons plus loin en détail, l'enseignement primaire et secondaire est une des compétences principales des collectivités locales et depuis la dévolution, il a été placé sous le contrôle du ministère écossais de l'Éducation (Scottish Executive Education Department). Le même transfert a été opéré au profit des institutions galloises. Le ministère chargé de l'Éducation au sein de Whitehall, à Londres, est donc uniquement en charge des établissements anglais ${ }^{9}$. En fait, l'enseignement primaire et secondaire a toujours été différent en Écosse, du fait que l'Acte d'Union de 1707 a imposé le maintien du système judiciaire écossais ainsi que la primauté de l'Église d'Écosse, d'obédience presbytérienne, qui est à l'origine d'un système scolaire lui-même spécifique.

Il n'existe donc pas de religion d'État au RoyaumeUni : l’Église anglicane est, comme son nom «Church of England» l'indique, la religion établie de la seule Angleterre, à l'exclusion explicite de l'Écosse et de l'Irlande du Nord, et implicite du Pays de Galles pour lequel rien n'a jamais été précisé officiellement.

Pour autant, depuis le règne d'Elizabeth I ${ }^{\text {re }}$, l'Église anglicane est placée dans une situation de dépendance par rapport aux organes de l'État: le souverain en est le chef et en nomme les principaux dignitaires ; la cérémonie du couronnement est une cérémonie religieuse ; toutes les séances du Parlement commencent par une prière; les deux archevêques et vingt-quatre des plus importants évêques siègent à la Chambre des Lords, dans l'attente du vote d'un projet de loi supprimant ce privilège. Toutefois,

2. J. Baubérot, « Géographie de la laïcité».

3. Ibid. Ainsi dans l'affaire Dahlab c. Suisse (15 février 2001), la décision de la Cour européenne des droits de l'homme vient opportunément rappeler que par les articles 164 et suivants de la Constitution cantonale de Genève, «ce canton connaît une séparation nette de l'Église et de l'État, au sens d'une laïcité de celui-ci».

4. O. Dord, «Laïcité : le modèle français sous influence européenne », Notes de la Fondation R. Schumann, L’Europe en actions, 2003.

5. Ibid., p. 14 .

6. Ibid., p. 28

7. En Irlande du Nord, le fonctionnement des institutions reste suspendu à la résolution politique du conflit entre Catholiques et Protestants, prévue par l'accord du Vendredi Saint signé par le Gouvernement Blair et tous les principaux partis politiques. Les élections législatives de mai 2005, qui ont vu le triomphe des idéologies les plus radicales dans les deux camps, auront probablement pour effet de retarder encore la dévolution pourtant approuvée par référendum en 1999 et appliquée pendant quelques mois.

8. Voir M. Breuillard, L'Administration locale en Grande-Bretagne, entre centralisation et régionalisation, Paris, L'Harmattan, 2000, p. 33-86.

9. Voir M. Breuillard et A. Cole, L'École entre État et collectivités locales, en Angleterre et en France, Paris, L'Harmattan, 2003. 
l'Église anglicane dispose de peu de privilèges par rapport aux autres Églises. L'État ne finance aucun culte, pas même le sien, mais toutes les communautés religieuses peuvent obtenir des aides indirectes sous la forme de subventions pour l'entretien de certains bâtiments, ainsi que le statut d'institution charitable qui leur permet de jouir d'un régime fiscal avantageux, notamment de l'exemption de l'impôt sur leurs revenus, sans bénéficier d'aucun privilège en matière de $\mathrm{TVA}^{10}$.

Elle a d'abord un devoir d'assistance: le système paroissial couvre tout le pays, s'applique à tous : chacun, quelle que soit sa confession, a accès au baptême, au mariage religieux et à l'office funèbre de l'Église anglicane de sa paroisse. Ce qui fait que les lieux de culte anglicans peuvent être considérés comme les derniers « services publics » présents dans les quartiers les plus défavorisés dont les prêtres assument une fonction de travail social autant qu'une mission pastorale ${ }^{11}$.

\section{B. La liberté religieuse, principe unique garanti par l'État}

Il n’a jamais semblé nécessaire aux Britanniques d'inventer un «droit des cultes» ni aucune législation dans ce domaine, l'État n'intervenant en aucune manière dans la pratique confessionnelle. Inimaginable que le Home Office, dont les attributions rappellent celles du ministère de l'Intérieur, agisse comme un "ministère des cultes»! Ayant pour seul rôle « de contribuer à l'instauration d'une société dans laquelle les différents systèmes de croyance, qu'ils soient religieux ou autres, constituent des valeurs comprises et respectées ", il ne se penche sur les questions religieuses qu'au travers de sa cellule « Faith Communities Unit» mise en place pour que « coopèrent le Gouvernement et les communautés confessionnelles ${ }^{12}$. Pour mieux connaître ces communautés confessionnelles, le recensement national de la population comporte une question relative à l'affiliation religieuse des habitants et permet ainsi de savoir que les Anglais se répartissent entre Chrétiens $(71,6 \%)$, Bouddhistes ( $0,3 \%)$, Juifs ( $0,5 \%)$, Musulmans $(2,7 \%)$, Sikhs $(0,6 \%)$ et d'autres religions $(0,3 \%)^{13}$. $\mathrm{Si}$, au total, $76,8 \%$ des résidents se disent appartenir à une religion, «le gouvernement a l'obligation d'assurer que ceux qui n'ont aucune croyance religieuse ne soient désavantagés en aucune façon ${ }^{14}$, ceux qui se disent n'appartenir à aucune religion $(15,5 \%)$ comme ceux qui n'ont pas répondu à la question $(7,3 \%)$.

Au nom de la tolérance, la liberté religieuse s'est progressivement imposée à la fin de la guerre civile dont les traumatismes ont longtemps marqué la mémoire collec- tive. Les protestants « non conformistes» (non anglicans) ont été émancipés dès 1689 , les papistes (catholiques romains) en 1829 et les juifs en 1858 . C'est ce principe qui a facilité l'implantation de multiples religions et sectes apportées par les émigrés, réfugiés politiques ou économiques, issus des pays du Commonwealth, des anciennes possessions coloniales et des pays en voie de développement.

En l'absence de constitution écrite, les principes fondamentaux relatifs à l'Église et à la religion restent ceux définis par les Bills of Rights du XVII ${ }^{\mathrm{e}}$ siècle, par quelques rares textes législatifs et par le droit coutumier. La liberté religieuse implique la liberté de prier, de s'exprimer et de mener sa vie conformément à ses croyances. Paradoxalement, l'Église anglicane fait figure d'exception en ce qu'elle est régie par la loi de 1534 qui fonde la religion née de la rupture d'Henry VIII avec la papauté. De nos jours, le Parlement doit encore ratifier les mesures prises par le Synode de l'Église anglicane mais le droit ecclésiastique, aujourd'hui clairement distinct du droit civil, ne figure plus dans les manuels de droit.

Si la laïcité est, selon Jean Baubérot, un «pacte [...], à la fois règlement juridique et art de vivre ensemble ${ }^{15}$, la liberté religieuse et la tolérance, son corollaire, sont des droits et libertés dont la jouissance appartient de facto à toute personne présente sur le sol britannique, sans qu'il soit nécessaire de les définir. Seul le Parlement peut éventuellement limiter cette jouissance s'il estime que l'intérêt général justifie la mise en œuvre de restrictions à l'exercice de ces droits et libertés.

Ainsi la France et la Grande-Bretagne se rejoignent dans le principe d'égalité entre les cultes : le respect de la liberté religieuse est inclus dans le respect de l'ordre public et toutes les religions ont droit d'expression. En fait ce qui les distingue l'une de l'autre, ce sont les conséquences tirées de l'affirmation de la liberté de chacun : en France, aucune religion ne doit accaparer l'État ni ne peut contredire les principes fondamentaux sur lesquels il repose ; en Grande-Bretagne, la législation comporte un important dispositif de lutte contre les discriminations sur le sexe, l'origine ethnique et le handicap, mais la discrimination religieuse n'est pas prohibée en tant que telle, sauf en Irlande du Nord. La loi refuse d'appréhender directement le domaine de la religion et c'est pourquoi, jusqu'à présent, les Britanniques ne comprennent pas qu'une loi ou un règlement puisse interdire l'expression d'aucune religion dans aucun lieu public, même à l'École, comme nous le verrons dans la troisième partie.

Le maitre mot est la tolérance, au nom de l'égalité et de la justice, là où le terme de neutralité domine en français.

10. Voir le Rapport sur le financement des communautés religieuses du Service des affaires européennes du Sénat, 2001.

11. Au début des années 1980, lors d'une série de révoltes dans les quartiers pauvres des grandes villes industrielles, les dignitaires de l’Église anglicane ont publiquement critiqué l'action du Gouvernement Thatcher et lancé un plan de financement de rénovation urbaine, pour obliger le gouvernement à assumer son devoir d'assistance envers les populations défavorisées.

12. Voir le rapport du groupe d'experts Steering Group reviewing patterns of engagement between Government and Faith Comminities in England : Working together: Co-operation between Government and Faith Communities, février 2004 (source: homeoffice.gov.uk).

13. National Census, 2001 (source : homeoffice.gov.uk).

14. Voir la présentation des compétences du Home Office sur le site officiel du Gouvernement britannique : homeoffice.gov.uk.

15. «Trois questions à... J. Baubérot », Regards sur l'actualité, $\mathrm{n}^{\circ}$ 298, février 2004, État, laïcité, religions. 
L'inexistence même du concept de laïcité en anglais se manifeste concrètement par la difficulté de traduire le substantif. Si en consultant les meilleurs dictionnaires anglais, on trouve «secularism", il faut bien admettre que ce terme évoque plutôt la philosophie du matérialisme. En désespoir de cause, la solution usuelle est de traduire « école laïque » par «non religious school» et « enseignement laïque » par «secular education».

Les relations entre la religion et l'école illustrent parfaitement les principes britanniques, sinon anglo-saxons, ainsi décrits et tracent une ligne de démarcation, probablement définitive, entre les deux visions du service public de l'enseignement.

\section{Enseignement public et liberté d'enseignement}

La gestion de l'enseignement primaire et secondaire est traditionnellement l'affaire des autorités locales. Le ministre ne s'autorise à intervenir qu'en cas de carence de l'autorité locale, constatée par un des membres du corps des inspecteurs de Sa Majesté. Dans le cadre général posé par la loi et les principes de liberté, la responsabilité des élus locaux est entière sur le plan financier, comptable, matériel et pédagogique.

Un établissement d'enseignement est public dans la mesure où il s'est soumis à la tutelle d'une autorité publique, en l'occurrence la collectivité locale compétente (Local Education Authority), ce qui lui assure un budget financé par des ressources essentiellement publiques. En effet, la réforme de 1944 que nous étudierons dans la seconde partie a permis d'intégrer au service public des établissements privés confessionnels, sous une forme plus ou moins contraignante et plus ou moins financièrement avantageuse, «selon que les organisations confessionnelles acceptaient ou non de tempérer leur caractère propre et leur doctrine particulière ${ }^{16}$. Ainsi des établissements fondés par les Églises anglicane, protestante, catholique et, plus récemment, musulmane, ont accepté de se soumettre au principe de la gratuité et de l'accès égal pour tous, en même temps qu'à la tutelle de l'autorité publique pour s'intégrer au service public de l'enseignement, tout en étant autorisé à conserver leur caractère confessionnel.

On peut certainement rapprocher cette conception de l'enseignement public britannique du projet du ministre Alain Savary d'intégration au service public de l'Éducation nationale des écoles privées sous contrat avec l'État. On sait que cette perspective a fait long feu sous la pression des syndicats de l'enseignement public qui ont fait monté les enchères jusqu'à un point qu'ils savaient inacceptable pour le secrétariat général de l'enseignement catholique. Il s'agissait pourtant d'aller jusqu'au bout de la logique du système initié par la loi Debré du 31 décembre $1959^{17}$.

Ainsi, la concurrence « à la française » entre « la Laïque» et "l'École libre», de même que le «mélange des genres » que constitue l'enseignement privé sous contrat, est impensable outre-Manche : la liberté d'enseignement permet l'existence d'établissements privés mais ceux-ci sont entièrement indépendants des autorités publiques, comme l'indique leur appellation «independent schools».

En application de la loi School Standards and Framework Act de 1998 modifiée en 2003, le ministère de l'Éducation et des Qualifications (Department for Education and Skills) contrôle et certifie le caractère religieux des établissements indépendants qui en ont fait la demande ${ }^{18}$. Sur les 2240 établissements privés, préélémentaires, élémentaires et secondaires existant en Angleterre, ont ainsi été reconnus en 2004: 9 musulmans, 2 à la fois orthodoxes et juifs, 4 méthodistes, 30 anglicans, 2 catholiques romains; 26 se déclarent simplement "chrétiens »; les autres se rattachent à des cultes divers : quaker, hernhute, adventiste, hindouiste ${ }^{19}$.

Cette certification les autorise à prendre en compte certaines considérations lors de la sélection de leur personnel, par exception aux règles de non-discrimination qui s'imposent à tout employeur. Elle constitue également la reconnaissance officielle de certains attributs de l'établissement scolaire et de son conseil d'administration.

Après avoir décrit le cadre institutionnel de l'enseignement public, il convient maintenant d'analyser le fonctionnement du «modèle» anglais de tolérance religieuse à l'École qui a évolué d'un enseignement des préceptes de la religion chrétienne à l'étude des religions.

\section{Le " modèle " anglais : de l'enseignement religieux à l'étude des religions}

En France, l'éducation est l'affaire de l'État depuis la Révolution: celle-ci ayant fait fuir une partie du clergé, l'État s'est imposé la tâche de remplacer l'Église catholique dans son quasi-monopole en matière d'éducation. C'est par l'école que la laïcité est entrée dans l'État. Au contraire, les souverains britanniques se sont attachés à faire de l'Église anglicane un instrument de promotion de la cohésion sociale et politique du royaume. La vision libérale d'un enseignement développé localement sous le contrôle vigilant du pouvoir central, qui était celle de la France du $\mathrm{XIX}^{\mathrm{e}}$ siècle jusqu'aux lois de Jules Ferry et à la loi de 1905, a perduré jusqu'à nos jours outre-Manche et, avec elle, la tradition de la pratique religieuse et de l'enseignement religieux en relation avec le caractère de chaque

16. Voir M. Lemosse, Le Système éducatif anglais depuis 1944, Paris, PUF (Perspectives anglo-saxonnes), 2000.

17. Voir B. Toulemonde, Petite histoire d'un grand ministère : l'Éducation nationale, Paris, Albin Michel, 1988.

18. Section 124 B nouvelle du chapitre 31 Independent Schools (Employment of Teachers in Schools with a Religious Character) Regulations 2003 (SI $2003 /$ 2037) et (SI 2003/2314).

19. Source: Statutory Instrument 2004 No.354 Designation of Schools Having a Religious Character (Independent Schools) England ( ${ }^{\circ}{ }^{2}$ ), Order 2004, 11 novembre 2004. 
établissement. Mais loin de l'instruction religieuse et de la direction spirituelle confiée aux aumôniers des lycées et collèges français, l'enseignement religieux en Angleterre est conçu comme un socle de valeurs communes et de connaissances qui s'est imposé dans le service public à travers l'enseignement religieux obligatoire, d'abord (A), l'enseignement des valeurs chrétiennes, ensuite $(C)$, à la suite du compromis politique que constitue la réforme de fond votée en 1944 (B).

\section{A. L'obligation de dispenser un enseignement religieux}

Pendant la période qui s'étend de la loi sur l'enseignement, qui généralise en 1872 l'enseignement primaire gratuit pour tous, et la réforme de 1944, qui définit le système scolaire en attribuant des compétences obligatoires et facultatives aux collectivités locales, seul l'enseignement religieux était mentionné comme matière obligatoire. Il faudra attendre 1988 et surtout 1998 pour que des directives ministérielles précisent le contenu des programmes scolaires, particulièrement précis en ce qui concerne les apprentissages fondamentaux (lecture, calcul et écriture) et inventent des évaluations de fin de cycle.

Avec l'industrialisation croissante, le besoin d'apprendre les rudiments de l'écriture, du calcul et de la lecture aux enfants des ouvriers s'est peu à peu imposé ${ }^{20}$ mais la question de l'instruction religieuse a longtemps divisé les pédagogues des deux principales organisations caritatives qui avaient ouvert des écoles, la National Society soutenue par l'Église anglicane, et la British and Foreign Schools Society des «non-conformists». Ces derniers, en particulier, s'opposaient à ce que des deniers publics puissent être accordés à des écoles relevant de l'Église établie.

En 1833, le Parlement réussit à imposer le versement de subventions aux sociétés philanthropiques ainsi qu'aux conseils de paroisse chargés d'ouvrir des écoles «publiques» (board schools) là où l'action des Églises et le nombre des church schools s'avéraient insuffisants, notamment dans les villes et villages devenus des centres industriels en plein expansion. Il ne s'agissait que de contribuer à l'entretien et à la construction des bâtiments, les enseignants étant rémunérés par leurs élèves. Les deux groupes d'établissements, board schools et church schools, étaient soumis au même contrôle du corps des Inspecteurs de Sa Majesté, créé en 1839, bien avant l'Inspection générale de l'Éducation nationale, en France. L'Église d'Angleterre et l'Église catholique furent les principales bénéficiaires de ce système de financement public puisqu'elles étaient aussi les plus actives. Il en ressort que, dès le départ, les écoles dispenseraient des enseignements religieux et pratiqueraient la prière en commun.

À partir de l'Education Act de 1872, la législation britannique a continuellement entériné la tradition d'une pratique religieuse et d'un enseignement religieux à l'école, tout en obligeant les établissements qualifiés de " publics » à accueillir des élèves de toute croyance d'une part, et à appliquer la clause de conscience, d'autre part. En application de cette clause de conscience, les parents peuvent retirer leurs enfants de l'école à l'heure des cours d'enseignement religieux et de la pratique religieuse. La clause de conscience s'entend également pour les enseignants qui sont autorisés à ne pas participer à la vie ou l'enseignement religieux de leur établissement.

Il est important de souligner que, pour conforter la vision « universaliste » de l'enseignement religieux, la loi de 1872 interdit aux établissements qui ne relèvent pas d'une Église de développer l'enseignement d'une foi religieuse particulière qui se réfère à une confession particulière.

Ainsi a été conçu le rapport entre l'école et les Églises : les écoles publiques sont obligées de dispenser un enseignement religieux mais ni les élèves ni les enseignants ne sont obligés d'y assister. C'est une conception tout à fait conforme à la vision libérale et anglo-saxonne des rapports entre la puissance publique et les individus : la première définit un cadre réglementaire mais les seconds sont libres en conscience d'y adhérer, dans la limite où le comportement individuel de chacun ne contredit pas les intérêts de la nation, dont le Parlement souverain est le gardien, in fine.

Au tournant du $\mathrm{xx}^{\mathrm{e}}$ siècle, lorsque les Églises ont commencé à trouver la charge scolaire financièrement trop lourde, elles ont envisagé de transférer leurs compétences à l'État. Cependant, elles s'inquiétaient du type d'enseignement qui serait délivré dans les écoles publiques. La solution satisfaisante qui permit de résoudre ce problème fut trouvée dans l'agrément de programmes d'enseignement religieux (agreed syllabus) c'est-à-dire proposés par les autorités locales compétentes à l'agrément des représentants religieux locaux.

À partir de 1924 le comté du Cambridgeshire s'est attelé à la préparation d'un programme d'enseignement religieux en concertation avec les confessions religieuses. Ce même programme fut rapidement adopté par d'autres autorités locales, pour respecter les équilibres locaux entre les différentes confessions présentes sur leurs territoires. L'Église anglicane accepta de plus en plus facilement le transfert de ses écoles aux collectivités locales lorsqu'un tel programme était mis en pratique. Par sa position d'Église établie, elle se considérait comme chargée pour la nation toute entière d'une responsabilité pastorale dont l'action éducative est une extension naturelle. À ses yeux, les établissements scolaires anglicans étaient accessibles à ceux qui appartiennent à l'Église anglicane mais aussi à ceux qui n'y appartiennent pas. Au contraire l'Église catholique, devenue minoritaire, s'est toujours sentie liée envers ses seuls membres. Son objectif était de s'assurer que ceuxci reçoivent un enseignement catholique délivré par des enseignants catholiques.

Ces deux conceptions divergentes ont pu être toutes les deux incorporées dans la grande réforme de 1944 qui

20. En 1816, l'industriel réformateur Robert Owen ouvre les premières écoles ( «infant schools») pour les enfants de ses employés et son exemple est suivi par quelques entrepreneurs inspirés par les écrits des Lumières. 
a mis en ordre de marche le service public de l'éducation garanti par l'État.

\section{B. Le compromis de 1944 : le service public diversifié}

Dès 1943, un rapport ministériel (Education Reconstruction), s'appuyant sur des sondages, déclarait qu' « il y avait un souhait général, non limité aux représentants des Églises, que l'enseignement religieux reçoive une place mieux définie dans la vie et le travail des écoles », eu égard au constat que « les quatre dictateurs de la Seconde Guerre mondiale avaient persécuté les églises et que le Christianisme renforçait la démocratie ${ }^{21}$. Tout en maintenant le système dual des board schools et church schools, la loi de 1944 leur a proposé deux modes d'intégration au service public: "county schools» (depuis 1998, «community schools») et « voluntary schools », déjà mentionnées. Désormais tous ces établissements définis comme étant «publics» (maintained schools), c'est-à-dire relevant de l'autorité des collectivités locales, sont traités de la même façon, dans le financement de leurs bâtiments, équipements et aussi de leur personnel, et obtiennent le même soutien de leur tutelle administrative. En contrepartie, ils doivent accueillir les mêmes publics dans le cadre de l'obligation scolaire, se soumettre au contrôle administratif et pédagogique de l'autorité locale de leur circonscription et respecter la réglementation nationale. Les établissements qui refusent les conditions d'exercice de cette mission de service public constitueront le secteur privé «indépendant».

Dans les établissements publics, l'enseignement religieux comporte les deux aspects mentionnés dans la loi : l'enseignement religieux et la prière commune. Ainsi l'enseignement religieux est dispensé dans tous les établissements sous contrôle des collectivités locales, tant les «voluntary schools» que les «county schools». Dans ces dernières, il devra respecter le programme agréé qui, nous l'avons vu, «ne contiendra aucun catéchisme ni dogme distinctif d'aucune confession religieuse particulière ${ }^{22}$. De nos jours, l'enseignement public compte trois catégories juridiques d'établissement: Community Schools, Voluntary Controlled Schools, et Voluntary Aided Schools, selon que la responsabilité de l'emploi du personnel, de l'inscription des élèves d'âge scolaire ou la propriété des terrains et bâtiments relèvent de la collectivité locale ou du conseil d'administration de l'établissement lui-même.

Ainsi l'on comprend que si les textes législatifs parlaient d'instruction religieuse, les déclarations de politique gouvernementale et tous les documents officiels accréditaient plutôt l'idée d'une "éducation religieuse ", voire d'une « éducation religieuse et morale» se référant alors exclusivement à l'héritage chrétien, commun à une société britannique considérée alors comme univoque, monolithique.

Cependant dans les années 1960, la mise en question des valeurs fondamentales de la société britannique fait écho au développement de religions non chrétiennes pratiquées par les immigrés dans les grands centres urbains. En outre, l'utilisation croissante de la clause de conscience par des parents se disant athées ou membres d'une religion non chrétienne, risquait de poser des problèmes insurmontables de logistique si les élèves devaient être autorisés à fréquenter un autre établissement scolaire pour la durée de leur pratique religieuse.

De multiples pressions ont été exercées sur le gouvernement pour réclamer une refonte des programmes agréés et une nouvelle définition de l'enseignement religieux. En outre, la politisation du débat sur la fonction sociale de la scolarisation, qu'ont connu la majorité des États de l'Europe occidentale à la même époque, s'est élargie en Grande-Bretagne à la question de l'impact de l'enseignement religieux sur cette fonction.

En effet, il est dit que chaque matière inscrite à l'emploi du temps des élèves doit pouvoir être justifiée par des critères éducationnels : elle doit participer à la formation d'une éthique personnelle ou bien au contraire à une exploration personnelle de la religion, dans le but de créer l'harmonie sociale et la coopération entre groupes sociaux et entre individus : l'enseignement de la religion a sa place dans un processus de démocratisation de l'enseignement en ce qu'il sert à produire plus de tolérance par une meilleure connaissance des divers groupes ethniques et religieux dont sont issus les élèves, amplifiant ainsi le brassage social dans des établissements plus diversifiés.

La religion est donc utile en tant que phénomène culturel.

Ce qui fait que si l'éducation reste considérée comme un moyen de faire avancer une certaine vision de la société, une majorité d'enseignants et d'hommes politiques se sont mis à envisager l'étude des religions, au-delà de la seule religion chrétienne.

\section{De l'enseignement religieux à l'enseignement des valeurs chrétiennes}

Après les querelles de spécialistes de la pédagogie autour des projets de réforme des Travaillistes des années soixante ${ }^{23}$, les partis politiques et l'électorat tout entier, audelà des parents et des enseignants, se sont emparés de la question de la qualité de l'enseignement public pour en faire un sujet de débat national. Le nombre inhabituel de réformes votées à l'initiative des gouvernements de Margaret Thatcher et de John Major, puis la place prise dans

21. Voir Religious Education in Great-Britain, Sweden and Russia. Presentations, Problem Inventories and Commentaries (PETER Project), E. Almén et H.C. Øster (dir.), Linköping Studies in Religion and Religious Studies, Linköping University Electronic Press, 2000 (accessible sur le site www.ep.liu.se/ ea/rel/200o/oo1/reloo1-contents.pdf).

22. Education Act, 1944, section 26.

23. Notamment les «comprehensive schools», proposées par R. Crossland, en 1968, qui préfigurent, mutatis mutandis, le Collège unique instauré en 1975 par René Haby. 
le programme électoral des Travaillistes pour les élections législatives de 1997, de 2001 et même de 2005, marquent une rupture avec la situation antérieure : les gouvernements conservateurs et travaillistes ont mis l'accent sur la préparation à la vie professionnelle et la nécessaire adéquation entre cette préparation et les besoins du marché de l'emploi.

Depuis les gouvernements conservateurs des années quatre-vingt, en effet, la politique éducative s'efforce de répondre à la question suivante : comment les établissements scolaires peuvent-ils contribuer à la création d'une économie plus rentable et plus compétitive? Ce lien établi par les politiques entre formation initiale et « employabilité » se manifeste jusque dans les dénominations du ministère: Department for Education and Employment (DfEE), puis, depuis 2001, Department for Education and Skills (DfES).

Mais la question de l'enseignement religieux n'a pas été abandonnée pour autant et reste liée à la conception même de l'Éducation.

C'est ainsi que la loi de réforme de l'Éducation de 1988 contient un programme scolaire national ${ }^{24}$, dont les objectifs visent au « développement spirituel, moral, culturel, mental et physique des élèves [...] et les prépare à répondre aux opportunités et aux expériences de la vie d'adulte». En ce qui concerne l'enseignement religieux, les programmes locaux agréés «doivent refléter le fait que les traditions religieuses en Grande-Bretagne sont en majorité chrétiennes tout en prenant en compte l'enseignement et les pratiques des autres religions principales représentées en Grande-Bretagne » ${ }^{25}$. La pratique de la prière commune dans les écoles devrait refléter « les grandes traditions de la croyance chrétienne sans signe distinctif d'aucune confession chrétienne particulière ${ }^{26}$.

Si l'Education Act de 1944 avait déjà prévu un enseignement des principes de la religion chrétienne et la pratique de la prière, les réformateurs de 1988 ont tenu à réaffirmer «la primauté de la chrétienté dans le patrimoine culturel anglais en tant que cadre de règles principales et nécessaires à la restauration des valeurs morales [...] et à la reconnaissance de l'importance du développement moral et spirituel pour les jeunes $\gg{ }^{27}$. Cela ne veut pas dire que la religion anglicane soit imposée à tous : la prière est définie comme «le respect ou la vénération dû à un être ou une puissance d'essence divine ${ }^{28}$.

Comme nous le verrons dans la partie suivante, les signes extérieurs religieux, même non chrétiens, comme le port du foulard islamique ou de la kippa juive, ne soulèvent aucune polémique tant qu'ils peuvent s'associer au port de l'uniforme scolaire, et les religions non chrétiennes ont été incorporées dans le programme d'étude des religions.

Chaque autorité locale compétente en matière d'enseignement est tenue de mettre en place un Standing Advisory Council on Religious Studies (SACRE) composé d'enseignants et d'élus locaux et de représentants locaux de l'Église d'Angleterre et des autres religions, chrétiennes ou non, présentes dans la communauté concernée. Rappelant des commissions mises en place dès 1944, les SACRE sont consultés notamment pour la préparation des règlements intérieurs des établissements, pour juger des problèmes de dispense de participation de toute ou partie de l'effectif d'un établissement à la prière commune ${ }^{29}$. Les parents insatisfaits de l'application de la loi peuvent recourir à une procédure de réclamation qui peut remonter $\mathrm{du}$ conseil d'établissement jusqu'au ministre lui-même ${ }^{30}$.

Tout en faisant de l'éducation sa priorité des priorités, depuis douze ans, le gouvernement Blair n'a en rien modifié cette approche consensuelle de la religion à l'école et c'est bien hors de toute considération partisane qu'il faut analyser l'affaire S. Begum, qui fait l'objet de notre troisième et dernière section.

\section{L'affaire Shabina Begum, défense et illustration du « modèle anglais "}

Par son origine même, l'affaire Shabina Begum démontre l'écart qui sépare les appréciations française et anglaise de la liberté d'expression de la confession religieuse à l'école car il est certain qu'en France l'affaire aurait commencé beaucoup plus tôt dans la chronologie des faits. Elle souligne également la différence d'approche du « dialogue avec l'élève » que l'on retrouve dans les lois françaises et anglaises et qui doit précéder la mise en œuvre d'une procédure disciplinaire.

Après avoir été admise à porter, à partir de l'âge de 12 ans et pendant plusieurs années le hijab (foulard, pantalon et tunique) à la place de l'uniforme traditionnel de son établissement secondaire de la région londonienne, la jeune Shabina Begum décida, à la rentrée scolaire de septembre 2002, donc trois ans plus tard, de porter une jellaba couvrant tout le corps à l'exception du visage et des mains, plus conforme aux préceptes de sa religion. La direction de l'établissement lui refusa ce changement vestimentaire. Exclue par décision du conseil de discipline, elle intenta un recours contre son école au motif que le refus de l'établissement contrevenait à son droit à l'éducation et à la liberté de religion telle qu'affirmée par la Convention européenne des droits de l'homme.

\footnotetext{
24. Le programme impose l'enseignement de 9 matières: anglais, sciences et mathématiques (les trois matières fondamentales auxquelles la langue galloise est ajoutée pour le Pays de Galles) et l'histoire, la géographie, la technologie, la musique, les arts plastiques et l'éducation physique.

25. Loi de 1988, section 8 (3).

26. Ibid., Section 7 .

27. Voir les débats parlementaires à la Chambre des Lords: Baroness Cox, Bishop of Truro, Hansard, février et juin 1988, cités par D. Cooper, «Defiance and Non-Compliance, Religious Education and the Implementation Problem », Current Legal Problem, vol. 48, 1995, p. 253 -279.

28. Education Reform Act, Circular $1 / 94$ (par. 57).

29. Education Reform Act, 1988, Schedule 11.

30. Education Reform Act, Circular 1 / 94 (par. 134-140). Voir D. Cooper, «Defiance and Non-Compliance...».
} 
En première instance, en juin 2004, elle fut déboutée: en l'autorisant pendant trois ans à porter le hijab, le collège, qui compte $80 \%$ d'élèves musulmans, avait fait la preuve qu'il appliquait une politique souple à l'égard de l'uniforme scolaire afin de respecter la religion et les sensibilités culturelles de chacun.

L'argumentation du représentant de l'établissement vaut d'être détaillée pour un lectorat français: Shabina n'a jamais été exclue mais s'est elle-même tenue à l'écart. La direction ne permet pas le port de la jellaba pour ne pas encourager certaines élèves dans l'idée qu'elles sont de «meilleures musulmanes » que celles qui ne portent que le foulard. Un autre argument, plus terre à terre, a été avancé, selon lequel le port d'une longue robe entraînait un risque de chute plus grand ${ }^{31}$.

Après avoir mené une active campagne médiatique pendant deux ans, défendue en appel par Cheerie Booth, l'épouse du Premier Ministre, la jeune Shabina a obtenu la cassation du jugement de première instance en mars $2005^{32}$. L'avocate a posé le problème de l'exclusion scolaire sous l'angle des droits fondamentaux: quelle est la nature du droit de l'élève à l'éducation et comment l'interpréter au regard de sa liberté d'expression religieuse?

Dans son arrêt, Lord Justice Brooke a affirmé que Denbigh High School avait nié le droit de Shabina Begum de manifester sa religion et, en même temps, le droit d'accéder à l'éducation scolaire qui lui convient puisque l'élève avait dû s'inscrire dans un autre établissement. Il a fait appel au ministère de l'Éducation pour qu'il donne aux établissements des directives plus claires sur la façon de respecter les obligations que leur impose la Convention européenne de sauvegarde des droits de l'homme et des libertés fondamentales que le Royaume-Uni a ratifiée en 1998.

Cet arrêt devrait avoir des répercussions importantes dans les nombreux établissements qui accueillent des enfants issus de groupes religieux divers. Déjà en juin 2004, un rapport à la Chambre des Lords demandait que les écoles publiques fassent des efforts pour mieux répondre aux besoins des élèves musulmans et souhaitait la création d'école confessionnelles plus nombreuses pour satisfaire à ces besoins ${ }^{33}$.

Là encore la réponse de Steve Sinnott, secrétaire général de National Union of Teachers, un des principaux syndicats des enseignants des établissements publics, est significative de la façon dont on pose le problème outreManche: «Je ne crois pas que la réponse soit: plus d'écoles confessionnelles. Les établissements publics doivent s'adapter aux besoins de leur communauté. C'est un constat d'échec s'il se développe plus d'écoles confessionnelles. Il est important que les écoles respectent les traditions religieuses de communautés représentées parmi leurs élèves. Il n'y a aucune raison pour que la couleur d'un uni- forme scolaire, par exemple, ne puisse être utilisée pour la jellaaba ». Sur la même ligne de pensée, la commission parlementaire chargée des collectivités locales (Office of the Prime Minister (ODPM) Select Committee) recommandait de réformer le programme scolaire pour tenir compte des besoins des musulmans : «les écoles confessionnelles ne devraient pas être autorisées à moins qu'elles ne fassent une promotion active du multiculturalisme». Son président, le député Andrew Bennett, rappelait que «l'Irlande du Nord a fait la démonstration des dangers des école établies sur les confessions $»^{34}$.

Par ricochet, l'affaire Begum remet incidemment en question le port de l'uniforme scolaire comme signe distinctif d'appartenance à un établissement scolaire spécifique, public ou privé, mais c'est une autre histoire...

\section{Conclusion}

Ainsi donc on voit se creuser le fossé entre deux interprétations d'une liberté fondamentale qui, a priori, est acquise depuis longtemps dans nos États d'Europe occidentale.

En France, la neutralité du service public est tirée du principe constitutionnel de laïcité inscrit dans la Déclaration des droits de l'homme et le préambule de la Constitution qui exclut par définition que les cultes puissent constituer une activité de service public. La laïcité est la neutralité confessionnelle du service public qui fait que les moyens du service ne peuvent véhiculer aucune croyance et de plus les agents chargés de son exécution ne peuvent exprimer leurs convictions dans l'exercice de leurs fonctions. Depuis la loi du 15 mars 2004, dont l'application est limitée à l'école publique et plutôt au territoire métropolitain, la neutralité imposée est étendue aux usagers du service public: «Dans les écoles, les collèges et les lycées publics, le port de signes ou tenues par lesquels les élèves manifestent ostensiblement une appartenance religieuse est interdit ${ }^{35}$.

L'Angleterre ne fait aucune distinction entre le service public et les autres activités humaines pour l'exercice de la liberté d'opinion et d'expression politique, philosophique ou religieuse, et s'en tient à une seule limitation : celle que la loi peut imposer dans les cas où il y a nécessité de sauvegarder les intérêts de la sécurité publique, de protéger l'ordre public, la santé ou la moralité ou les droits et libertés d'autrui. C'est pourquoi il n'y a pas de difficulté à accepter, par exemple, la transformation des uniformes, celui d'une femme musulmane, membre de la police, en ajoutant un voile au képi, celui d'une élève musulmane, en transformant la tenue traditionnelle (jupe et blazer) en pantalon-tunique, en acceptant le port d'un turban pour les élèves de religion sikh, du moment que le signe

31. Voir l'article de S. Jones, «Muslim pupil lose legal battle to wear jilbab », The Guardian, 16 juin 2004.

32. Voir Shabina Begum v. Denbigh High School (Luton), mars 2005.

33. Rapport à la Chambre des Lords, 9 juin 2004

34. Voir la note de Press Association, 18 février 2005

35. Article L 141-5-1 du Code de l'Éducation, issu de la loi no 2004-228 du 15 mars 2004, art. 1, JO du 17 mars 2004, en vigueur le $1^{\text {er }}$ septembre 2004. 
considéré comme le plus important, la couleur de l'uniforme et / ou son allure générale, n'est pas modifiée au point de perdre sa signification aux yeux des tiers.

Cette affaire exprime, à notre avis, l'idée toute britannique que la tolérance religieuse, c'est à la fois le droit à la différence et à la manifestation de sa différence, dans la religion comme dans tous les actes de la vie privée. Et la religion relève de la sphère privée, en Grande-Bretagne!

Dans un pays de religion établie qui impose un enseignement des valeurs chrétiennes au cœur de son enseignement public, l'affaire Begum vient affirmer, une fois encore, que l'identité religieuse des musulmans peut s'exprimer tant que les valeurs musulmanes ne portent pas atteinte à l'ordre public et ne contreviennent pas aux valeurs chrétiennes qui inspirent les principes fondamentaux du royaume. On est loin du projet républicain d'intégration à la française qui veut gommer toute marque d'identité religieuse à l'école, « tout signe d'appartenance religieuse ostentatoire »! La loi du 15 mars 2004 est bien, comme l'a exprimé l'archevêque de Canterbury, une loi «impensable en Grande-Bretagne ».

On comprend mieux pourquoi les Anglais, comme d'autres Européens, d'ailleurs, souhaitaient faire inscrire dans le projet de constitution européenne la référence à l'importance de l'héritage chrétien pour la civilisation européenne... et combien les Français qui s'en sont insurgés se sont trompés de cible en réclamant une constitution laïque! L'enseignement de la religion est censé contribuer à la conception que l'éducation est un des véhicules qui font progresser la société, celle de Grande-Bretagne comme celles des pays nordiques, tous historiquement protestants ${ }^{36}$. Si Tony Blair, lors d'une séance à la Chambre des Communes, a indiqué qu' « il souhaitait que les Églises de Grande-Bretagne jouent un plus grand rôle dans la vie de la nation », il a aussi précisé qu'en aucun cas il ne voulait voir la Grande-Bretagne tomber dans la bigoterie et «copier les États-Unis où tout le monde se bat la coulpe à propos de sa religion ${ }^{37}$.

Comme souvent dans les comparaisons franco-britanniques, les problèmes sont les mêmes mais leurs solutions diffèrent. Â propos de l'enseignement religieux à l'école, c'est une opposition points par points, entre le RoyaumeUni, de religion établie, et la France, république laïque, qui amène la réponse à la question: dans quelle mesure un enseignement non confessionnel sur la religion devrait contribuer, ou remet en question, la façon dont un élève comprend sa tradition religieuse et dans quelle mesure ces questions devraient être réservées à l'enseignement confessionnel ${ }^{38}$.

$\mathrm{Si}$ «le modèle français de laïcité sert de repoussoir » au Royaume-Uni ${ }^{39}$, l'évocation du «communautarisme anglo-saxon » amène, trop souvent en France, à des anathèmes qui relèvent plus de l'approximation que d'une réelle connaissance au fond. Une fois encore, on attend une prise de conscience de l'intérêt des études comparatives et de la nécessité de développer l'enseignement du droit comparé «autrement que comme une sorte de coquetterie intellectuelle dépourvue d'intérêt pratique » que fustige le Professeur Lichère ${ }^{40}$ !

\footnotetext{
36. Voir Religious Education in Great-Britain, Sweden and Russia. Presentations, Problem Inventories and Commentaries.

37. Voir l'article de Matthew Tempest, correspondant politique, «Blair calls on churches to play "bigger role” ", The Guardian, 22 mars 2005.

38. Religious Education in Great-Britain, Sweden and Russia. Presentations, Problem Inventories and Commentaries, p. 126.

39. Voir J. Baubérot, « Géographie de la laïcité».

40. F. Lichère, « Du droit comparé et du droit tout court », AJDA, 2004, Chroniques, p. 2017.
} 